\title{
Retinal thickness and microvascular alterations in the diagnosis of systemic lupus erythematosus: a new approach
}

\author{
Ren Liu ${ }^{1}$, Yan Wang ${ }^{1}$, Qiang Xia ${ }^{1}$, Tian Xu ${ }^{1}$, Ting Han ${ }^{1}$, Shuang Cai ${ }^{1}$, Shui-Lin Luo ${ }^{1}$, Rui Wu ${ }^{1}$, Yi Shao ${ }^{2}$ \\ ${ }^{1}$ Department of Rheumatology, The First Affiliated Hospital of Nanchang University, Nanchang, China; ${ }^{2}$ Department of Ophthalmology, The First \\ Affiliated Hospital of Nanchang University, Jiangxi Province Ocular Disease Clinical Research Center, Nanchang, China
}

Contributions: (I) Conception and design: Y Shao, R Wu; (II) Administrative support: Y Shao, R Wu; (III) Provision of study materials or patients: R Liu, Y Wang, Q Xia, T Xu; (IV) Collection and assembly of data: R Liu, T Han, S Cai, SL Luo; (V) Data analysis and interpretation: R Liu, Y Shao, R Wu; (VI) Manuscript writing: All authors; (VII) Final approval of manuscript: All authors.

Correspondence to: Rui Wu. Department of Rheumatology, The First Affiliated Hospital of Nanchang University, 17 Yongwaizheng Street, Donghu District, Nanchang 330006, China. Email: tcmclinic@163.com; Yi Shao. Department of Ophthalmology, The First Affiliated Hospital of Nanchang University, No 17, Yongwaizheng Street, Donghu District, Nanchang, China. Email: freebee99@163.com.

Background: To investigate the differences of retinal thickness (RT) and superficial vascular density (SVD) between patients with systemic lupus erythematosus (SLE) and healthy controls using optical coherence tomography angiography (OCTA).

Methods: Individuals with SLE ( $\mathrm{n}=12 ; 24$ eyes) and healthy controls ( $\mathrm{n}=12 ; 24$ eyes) were recruited to this study. The study protocol was in accordance with the tenets of the Declaration of Helsinki (as revised in 2013). The monocular best-corrected visual acuity (BCVA) was determined using a Snellen eye chart. Each image was segmented into 9 early treatment diabetic retinopathy study subregions, within which the macular RT and SVD were measured by OCTA. The vascular perfusion area as a percentage of the measured area was considered to be the vascular density.

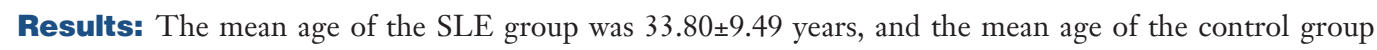
was 33.20 \pm 9.41 years. The mean duration of SLE was $4.33 \pm 2.67$ years. The BCVA was significantly different between the SLE and control groups $(0.17 \pm 0.20$ vs. $0.05 \pm 0.07$, respectively; $\mathrm{P}=0.021)$. In the SLE group, inner RT was reduced in the outer superior and temporal regions and full RT was reduced in the outer temporal region, compared with the control group $(\mathrm{P}<0.05)$. In the outer temporal region, the area under the receiver operating characteristic curve (AUC) for the inner RT was 0.805 [95\% confidence interval (CI): 0.674 to 0.935 ], and the full RT was 0.828 (95\% CI: 0.701 to 0.955 ). Thinning of RT was negatively correlated with erythrocyte sedimentation rate (ESR) in the inner retina at the outer temporal and outer superior regions and the full retina at the outer temporal region $(\mathrm{P}<0.05)$. The SVD was significantly lower in SLE participants than in controls in the central region, all 4 inner quadrants, and 4 outer quadrants $(\mathrm{P}<0.05)$. In the SLE group, SVD was positively correlated with inner RT in the outer superior region, inner RT, and full $\mathrm{RT}$ in the outer temporal region $(\mathrm{P}<0.05)$.

Conclusions: Variations in RT within the macular area may affect visual acuity. The OCTA measurement of RT may be a potential marker for diagnosis of SLE and an indicator of its inflammatory activity.

Keywords: Retinal thickness; vascular density; systemic lupus erythematosus (SLE); diagnosis; new approach

Submitted Apr 01, 2021. Accepted for publication Aug 05, 2021.

doi: 10.21037/qims-21-359

View this article at: https://dx.doi.org/10.21037/qims-21-359

\footnotetext{
^ ORCID: 0000-0001-8967-130X.
} 


\section{Introduction}

Systemic lupus erythematosus (SLE) is a common, longterm rheumatic disease, which affects about 20-150 per 100,000 people (1), $90 \%$ of whom are women of childbearing age (2). Any organ of the human body can be impacted by SLE, including the eyes (3), and its clinical manifestations vary greatly, creating challenges in its diagnosis and treatment by clinicians. The condition also has a major impact on patients' quality of life and has a 10 -year survival rate of about $70 \%$ (4).

Most patients with SLE have occult onset and a period of about 6 months to 4 years from the first symptom to diagnosis. In the early stage, it has many clinical manifestations that are common to other diseases, leading to misdiagnosis and missed diagnosis. The following clinical indicators suggest a need for close follow-up for suspected SLE: (I) clinical symptoms meeting the diagnostic criteria of SLE; (II) noninfectious fever; (III) leukopenia, hemolytic anemia, or hypocomplementemia; (IV) presence of multiple autoantibodies; (V) positive for anti-double-stranded DNA; (VI) positive for anticardiolipin antibody; and (VII) gradual increase in clinical manifestations and autoantibodies (5).

With the aim of facilitating a comprehensive evaluation and early diagnosis of SLE, the American College of Rheumatology (ACR) updated its itemized standard for SLE (6), including clinical and immune evaluation. The new classification does not include ocular indications. However, recent studies have shown that eye symptoms may reflect systemic disease activity and may manifest in one-third of SLE patients (3). In SLE, there may be involvement of the eyelids, orbit, ocular appendages, sclera, cornea, retina, uvea, optic nerve, and other ocular structures. Keratoconjunctivitis sicca (dry eye), retinal and choroidal vasculitis, and changes in the optic nerve are ocular manifestations of SLE (7), and the incidence of retinal involvement is $7-26 \%$ (8). Retinopathy is an accurate reflection of SLE activity in both recessive and dominant cases, and SLE patients with related retinopathy have been found to have a significantly lower survival rate than those without retinopathy (9).

Currently, fluorescein angiography (FA) and indocyanine green angiography (ICGA) remain the most important techniques for the exploration of fundus vascular diseases. However, both are invasive procedures that involve the infusion of contrast agent, which entails a risk of leakage affecting the evaluation of retinal vessels $(10,11)$. Optical coherence tomography angiography (OCTA) offers a noninvasive and clinically feasible retinal imaging method with higher image resolution than that of FA or ICGA (12). This technique allows measurement of fine structures in the retina and choroid (13-16) and has advantages in the diagnosis of disease, including optic neuritis $(\mathrm{ON})(17)$, Alzheimer's disease (18), diabetic retinopathy (19), thyroidrelated ophthalmopathy (20), and Parkinson's disease (21). Peripheral retinal nerve fiber layer (pRNFL) thinning, especially in the temporal region, has been recognized as a biomarker of neurodegeneration (22). Some previous studies have found similar findings in SLE patients. Macular RNFL and thickness may be a useful biomarker of early retinal and/or central nervous system (CNS) involvement in SLE (23). Photoreceptor layer and pRNFL thinning are early signs of retinal neurodegeneration in SLE (24). Patients with SLE have chronic low-grade CNS inflammation, which begins early in the course of the disease (25). The retina has the same embryonic origin as the section of the brain in charge of cognition. It has a similar blood-tissue barrier to the brain and contains unmyelinated axons, and is thus an ideal structure to observe brain changes and neurodegeneration (26). These characteristics suggest that ocular changes may provide valuable evidence for the early screening of SLE.

At present, OCTA is not widely used in SLE, and the findings of related research have been controversial $(23,27)$. Liu et al. (23) found that compared with a control group, SLE patients showed significant RNFL and macular thinning. However, Shulman et al. (27) found no significant difference in RNFL thickness between SLE patients and control group participants. Among the eye changes of SLE, immunoglobulin $\mathrm{G}(\mathrm{IgG})$ immune complex has been found in the retinal vascular wall, and this has been associated with RNFL infarction and ganglion cell atrophy (28). Vasculitis leading to retinal micro infarction could be the cause of RNFL and macular thinning in SLE patients. Therefore, in the present study, we investigated the ocular status of patients with SLE, and used OCTA to assess the retinal thickness (RT) and vascular density of SLE patients through comparison with healthy controls.

\section{Methods}

\section{Participants}

This cross-sectional study was conducted in 2020 at the Department of Ophthalmology and Rheumatology of the First Affiliated Hospital of Nanchang University 
(Nanchang, China). Patients with SLE were recruited from the Outpatient Department of Rheumatism Immunology, and gender- and age-matched controls were recruited from the Ocular Disease Clinical Research Center. The normal control group consisted of healthy participants, and an ophthalmologist from the medical center evaluated the absence of abnormalities in the eyes of these participants through clinical examination and OCTA imaging. All participants were examined by the same retinal specialist.

\section{Recruitment criteria}

All patients met the ACR criteria for SLE as revised in 1997 (29). The patients were aged between 18 and 60 years and had no signs or prodromal symptoms of lupus retinopathy, choroidopathy, or optic neuropathy. These criteria excluded patients with hydroxychloroquine (HCQ) toxicity-related chorioretinopathy.

\section{Exclusion conditions}

Individuals satisfying any of the following criteria were excluded from the study: (I) autoimmune disease other than SLE; (II) systemic diseases, such as endocrine or nervous system disease that affect the eye or optic nerve; (III) retinopathy or choroidal disease (such as vascular occlusion, age-related macular degeneration, glaucoma, or high intraocular pressure); (IV) eye tumor, history of eye surgery, or trauma; (V) diseases with significant effects on fundus imaging; (VI) pregnancy or lactation; or (VII) contraindications, allergies, or intolerance to local anesthetics or mydriatic drugs.

\section{Ethical considerations}

All research methods were approved by the Medical Ethics Committee of the First Affiliated Hospital of Nanchang University (cdyfy2016023) and were conducted in accordance with the Declaration of Helsinki (as revised in 2013). The purpose, method, and potential risks were explained to all participants, who then signed an informed consent form.

\section{Clinical examinations}

All participants underwent the following clinical and ophthalmic examinations: (I) antinuclear antibody and antiphospholipid antibody testing; (II) erythrocyte sedimentation rate (ESR) and C-reactive protein (CRP) tests as indicators of inflammation; (III) SLE Disease
Activity Index 2000 (SLEDAI-2K) (30) and Systemic Lupus International Collaborating Clinics/ACR Damage Index (SDI) (31) for disease damage assessment; and (IV) eye and vision measurements, including visual acuity (Snellen chart) and intraocular pressure (Goldmann tonometry); (V) OCTA.

\section{OCTA}

We used RTVue Avanti XR system (Optovue, Fremont, CA, USA) to perform OCTA imaging to simultaneously display retinal cross section and microvessels. The scanning speed was set as 70,000 a-scans per second, the axial resolution was $5 \mathrm{~mm}$, the horizontal resolution was $22 \mu \mathrm{m}$, the central wavelength was $840 \mathrm{~nm}$, and the bandwidth was $45 \mathrm{~nm}$. A b-scan (along the $\mathrm{x}$-axis) in a $3 \mathrm{~mm} \times 3 \mathrm{~mm}$ scan pattern with 5 repetitions of angiography was used to image at 216 raster positions (along the $y$-axis), focusing on the fovea, and the acquisition time was 3.9 seconds. We captured a 1,080 b-scan (216 y position $\times 5$ position) at 270 frames per second (32). We obtained a $3 \mathrm{~mm} \times 3 \mathrm{~mm}$ OCTA image through a series of 4 volume scans using 2 horizontal and 2 vertical rasters (933,120 a-scans in total). A $3 \mathrm{~mm} \times 3 \mathrm{~mm}$ en face OCTA angiographic image was calculated for each eye.

After scanning, each retina was segmented into 9 Early Treatment Diabetic Retinopathy Study (ETDRS) (33) subregions, composed of 3 concentric rounds $(0.5,1.5$, and $3 \mathrm{~mm}$ in radius), and their thickness was analyzed. Each layer of the retina covered the (I) inner retina-from the internal limiting membrane (ILM) to the inner plexiform layer (IPL) - and (II) the full retina-from ILM to retinal pigment epithelium (RPE). We defined outer RT as the difference between full RT and inner RT. The vascular perfusion area as a percentage of the measured area was considered to be vascular density. The vascular density was determined with the threshold method, in which 2-dimensional en face images of superficial retina (the layer between the vitreous retinal interface and the front boundary of the ganglion cell layer) were created. The value of the image block was determined and assigned to each pixel perfusion (1) or background (0). The average value of the skeleton plate in the region of interest was scaled based on the pixel size $(512$ pixels $/ 3 \mathrm{~mm})$ to calculate vascular density from the center of the macula to the edge of the $3 \mathrm{~mm} \times 3 \mathrm{~mm}$ brightness gradient image. Macular RT and superficial vascular density (SVD) were measured. In all participants, the right eye was assessed first. Data from the left eye were flipped to obtain a mirror image of the right 
eye. Left and right eye data were averaged and analyzed together (Figure 1A).

\section{Statistical analysis}

The data were analyzed using SPSS version 22.0 (IBM Corp., Armonk, NY, USA) and GraphPad Prism version 8 (GraphPad Software, La Jolla, CA, USA), and are reported as mean \pm standard deviation. Independent samples $t$-test, chi-square test, and Fisher's exact test were used to compare data between groups. The generalized estimation equation was employed to compare RT and SVD between SLE eyes and control eyes, and data were adjusted for known confounding variables and to account for the correlation between eyes from the same individual. False discovery rate (FDR) was used to adjust $\mathrm{P}$ values. The relationship between RT and systemic and ocular variables was analyzed using univariate and multivariate regression analyses. Linear correlation analysis was conducted between RT (full thickness, inner layer, and outer layer) and both ESR and SVD in each group. To analyze the distinction between healthy and SLE participants, receiver operating characteristic (ROC) curves for RT (full, inner, and outer) and SVD were plotted. A P value $<0.05$ was deemed statistically significant.

\section{Results}

\section{Participants}

A total of 12 participants ( 24 eyes) were included in each group. The groups were statistically similar in age (the mean age of SLE was $33.80 \pm 9.49$ years, and the mean age of the control was $33.20 \pm 9.41$ years; $\mathrm{P}=0.881$ ) and gender ratio (11 females and 1 male in each group; $\mathrm{P}=1.0$ ). In the SLE group, the mean time since diagnosis was $4.33 \pm 2.67$ years, the mean SLEDAI-2K was 4.25 (range, $0-10$ ), and the mean SDI was 0.17 (range, $0-1$ ). The visual acuity of the SLE group was significantly lower than that of the control group $(\mathrm{P}=0.021$; Table 1).

\section{Analysis of the macular RT}

The subregional RT in the SLE and control groups is shown in Table 2 and Figure 1B. After adjustments were made for age, gender, intraocular pressure, visual acuity, and blood pressure, as well as for $\mathrm{P}$ values after multiple correction, the inner RT was found to be significantly lower in SLE patients than in controls in the superior and temporal quadrants of the outer ring $(\mathrm{P}=0.045$ and $\mathrm{P}=0.012$, respectively; Figure 1C). The other 2 regions of the outer ring (nasal: $\mathrm{P}=0.173$; inferior: $\mathrm{P}=0.081$ ), 4 regions of the inner ring (superior: $\mathrm{P}=0.123$; nasal: $\mathrm{P}=0.068$; inferior: $\mathrm{P}=0.131$; temporal: $\mathrm{P}=0.058)$, and foveal center $(\mathrm{P}=0.162)$ were not significantly different between the groups.

After multiple correction, there was no significant difference in outer RT between SLE participants and their healthy controls (Figure 1D). All 4 regions of the outer ring (superior: $\mathrm{P}=0.349$; nasal: $\mathrm{P}=0.126$; inferior: $\mathrm{P}=0.512$; temporal: $\mathrm{P}=0.102$ ), all 4 regions of the inner ring (superior: $\mathrm{P}=0.632$; nasal: $\mathrm{P}=0.701$; inferior: $\mathrm{P}=0.608$; temporal: $\mathrm{P}=0.340)$, and foveal center $(\mathrm{P}=0.733)$ showed no significant difference.

The full RT in SLE participants was significantly thinner than that of the control group in the temporal region of the outer ring $(\mathrm{P}=0.018$; Figure $1 E)$. No significant difference between groups was found in any of the other regions $(\mathrm{P}>0.09)$.

Univariate regression analysis showed a negative correlation between macular RT and visual acuity $(b=-6.373$; $\mathrm{P}=0.002$ ), but not for age, gender, intraocular pressure, or blood pressure. Multivariate regression showed a significant association between poor visual acuity $(\mathrm{b}=-7.271 ; \mathrm{P}=0.010)$ and thinner macular RT (Table 3).

\section{Analysis of the macular retinal superficial vessel density}

The SVD at different retinal subregions in the SLE and control groups are shown in Table 4 and Figure 1B. After adjustments were made for age, gender, intraocular pressure, visual acuity, and blood pressure, SVD was found to be significantly lower in SLE participants than in the controls on the nasal side (inner ring: $\mathrm{P}=0.004$; outer ring: $\mathrm{P}=0.003$ ), inferior (inner ring: $\mathrm{P}=0.003$; outer ring: $\mathrm{P}=0.003$ ), temporal (inner ring: $\mathrm{P}=0.003$; outer ring: $\mathrm{P}=0.002$ ), superior (inner ring: $\mathrm{P}=0.005$; outer ring: $\mathrm{P}=0.013)$, and center regions $(\mathrm{P}=0.002$; Figure $1 F)$.

\section{ROC analysis of inner, full RT, and superficial vessel density}

The OCTA data were analyzed to find the specificity and sensitivity of RT and SVD as diagnostic indicators for SLErelated changes (Figure 2). Significant differences between groups were found in the inner outer superior (OS), inner outer temporal (OT), and full OT in the SLE group. The 
A
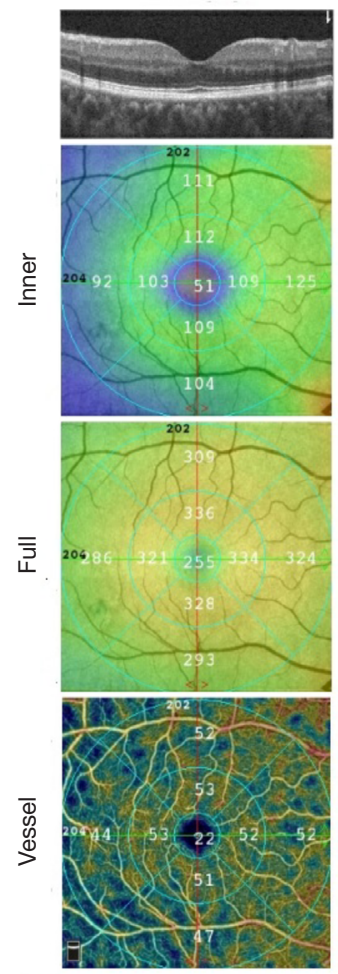

C$$
\text { C }
$$

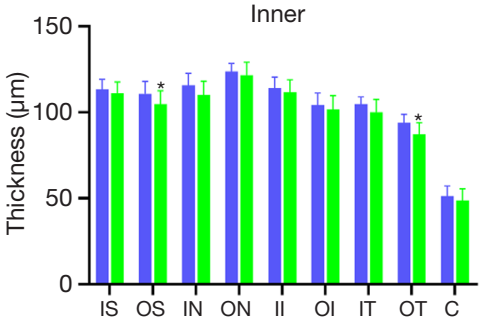

Control

- SLE

E

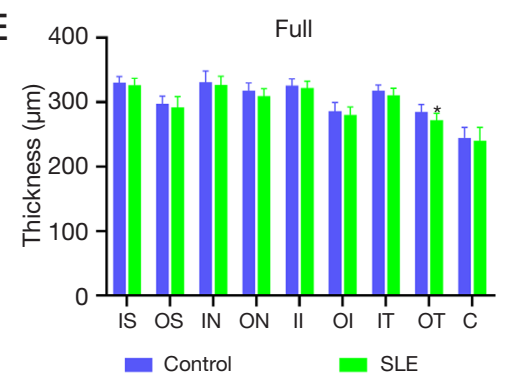

SLE
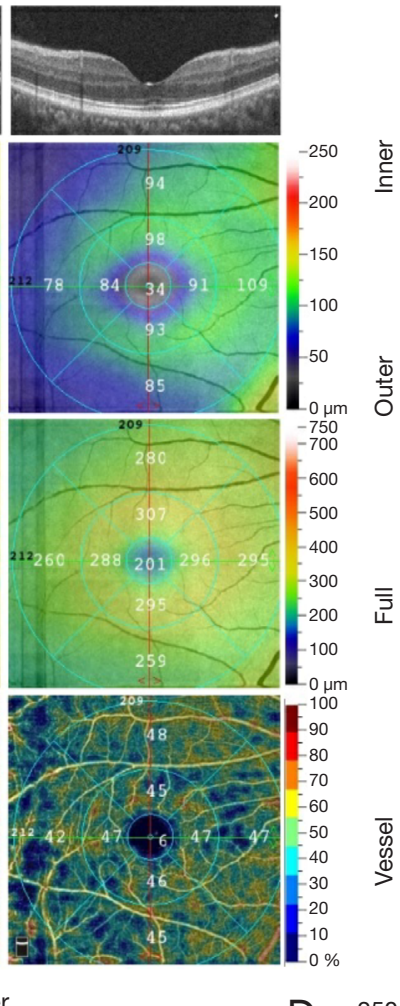

D 250

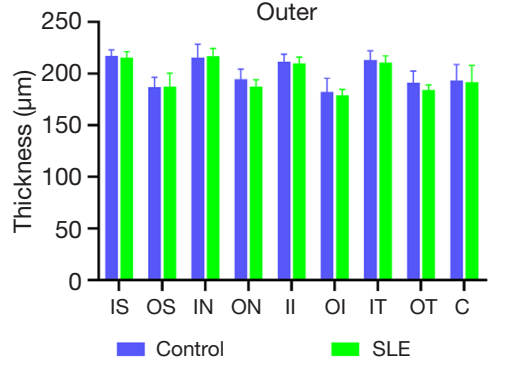

F

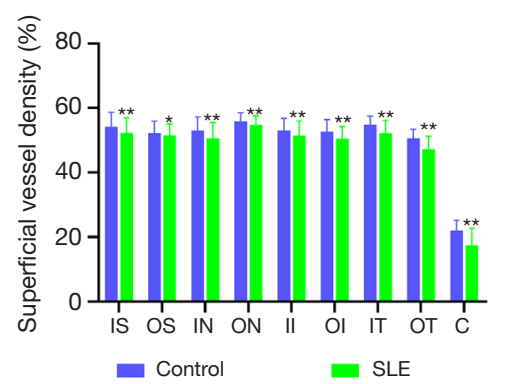

B

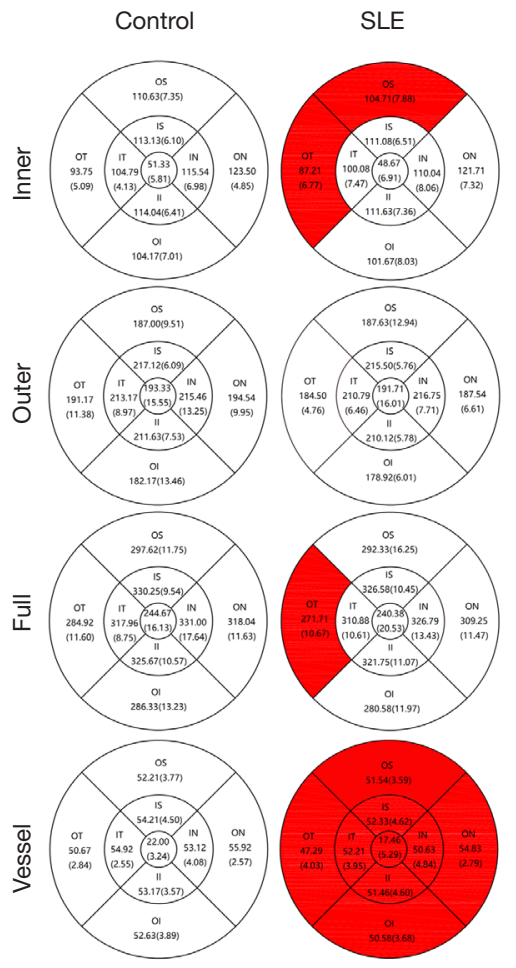

Outer 
Table 1 Characteristics of patients with SLE and healthy controls

\begin{tabular}{|c|c|c|c|c|}
\hline Characteristics & SLE ( $n=12 ; 24$ eyes) & $\mathrm{HC}(\mathrm{n}=12 ; 24$ eyes $)$ & $t$ & $P$ value \\
\hline Gender (female:male) & $11: 1$ & $11: 1$ & - & $1.000^{\mathrm{b}}$ \\
\hline Acuity (logMAR), mean \pm SD & $0.17 \pm 0.20$ & $0.05 \pm 0.07$ & - & $0.021^{\star c}$ \\
\hline Mean intraocular pressure $(\mathrm{mmHg})$, mean $\pm \mathrm{SD}$ & $16.04 \pm 2.68$ & $15.13 \pm 1.61$ & - & $0.253^{c}$ \\
\hline CRP (10 mg/L), mean \pm SD & $6.96 \pm 17.86$ & N/A & - & - \\
\hline ANA, n (\%) & $12(100.0)$ & N/A & - & - \\
\hline aPL, n (\%) & $1(8.3)$ & N/A & - & - \\
\hline SLEDAI-2K, mean (range) & $4.25(0-10)$ & N/A & - & - \\
\hline $\mathrm{DBP}(\mathrm{mmHg})$, mean $\pm \mathrm{SD}$ & $78.08 \pm 8.57$ & $82.83 \pm 6.98$ & -1.489 & $0.151^{\mathrm{a}}$ \\
\hline Duration of SLE (years), mean \pm SD & $4.33 \pm 2.67$ & $\mathrm{~N} / \mathrm{A}$ & - & - \\
\hline
\end{tabular}

*, $\mathrm{P}<0.05 ;{ }^{a}$, independent samples $t$-test; ${ }^{\mathrm{b}}$, Chi-square test; ${ }^{\mathrm{c}}, \mathrm{P}$ value was obtained with a generalized estimating equation (both eyes of the participants were included). SLE, systemic lupus erythematosus; HC, healthy control; SD, standard deviation; MAR, minimum angle of resolution; ANA, antinuclear antibody; aPL, antiphospholipid antibodies; SLEDAI-2K, SLE Disease Activity Index 2000; SDI, SLE Damage Index; ESR, erythrocyte sedimentation rate; CRP, C-reactive protein; N/A, not applicable; SBP, systolic blood pressure; DBP, diastolic blood pressure.

area under the ROC curve (AUC) for inner RT and full RT in the OT region was 0.805 (95\% CI: 0.674 to 0.935 ) and 0.828 (95\% CI: 0.701 to 0.955$)$, respectively, suggesting moderate to high diagnostic sensitivity for SLE (Figure $2 A$ ).

Significant between-groups differences in SVD were found in all 4 regions and in the central region. The AUC of the SVD OT was 0.779 (95\% CI: 0.644 to 0.913 ), and the AUC of the SVD center was 0.780 (95\% CI: 0.643 to $0.916)$, suggesting moderate diagnostic sensitivity of SVD for SLE (Figure 2B).

\section{Relationship between RT and ESR and CRP}

In patients with SLE, negative correlations were found between RT and ESR in the inner retina at OT and OS regions $(-0.456$ and -0.415 , respectively) and the full retina at OT (-0.478; Figure 3). There was no correlation between RT and $C R P$ in the inner retina at $\mathrm{OT}$ and $\mathrm{OS}$ regions $(\mathrm{P}=0.437$ and $\mathrm{P}=0.395$, respectively) and the full retina at $\mathrm{OT}(\mathrm{P}=0.153)$.

\section{Relationship between $R T$ and superficial vessel density}

As shown in Figure 4, in the SLE group, the correlation coefficient between inner RT and SVD in the OS region was 0.683 , that between inner RT and SVD in the OT region was 0.693 , and that between full RT and SVD in the OT region was 0.524 . These findings suggest that retinal thinning may be related to decreased vascular density in SLE.

\section{Discussion}

This study found significantly reduced visual acuity, RT, and retinal vascular density in patients with SLE, as well as significant associations between these factors.

The basic pathology of SLE involves vasculitis and its characteristic histological change is the deposition of immune complex, which especially affects the small arteries and capillaries. In the 2017 ACR classification standard for SLE (6), antinuclear antibody titer $\geq 80$ (Hep-2 cell immunofluorescence method) is emphasized as a necessary condition, and it is applied to 7 clinical (fever, skin, joint, nervous system, serositis, blood system, kidney) and 3 immunologic (antiphospholipid antibody, complement, and anti-Smith antibody) factors. Each criterion in the standard is stratified and weighted, and a total score of $\geq 10$ allows a 
Table 2 Comparison of macular retinal thickness at different locations between patients with SLE and healthy controls

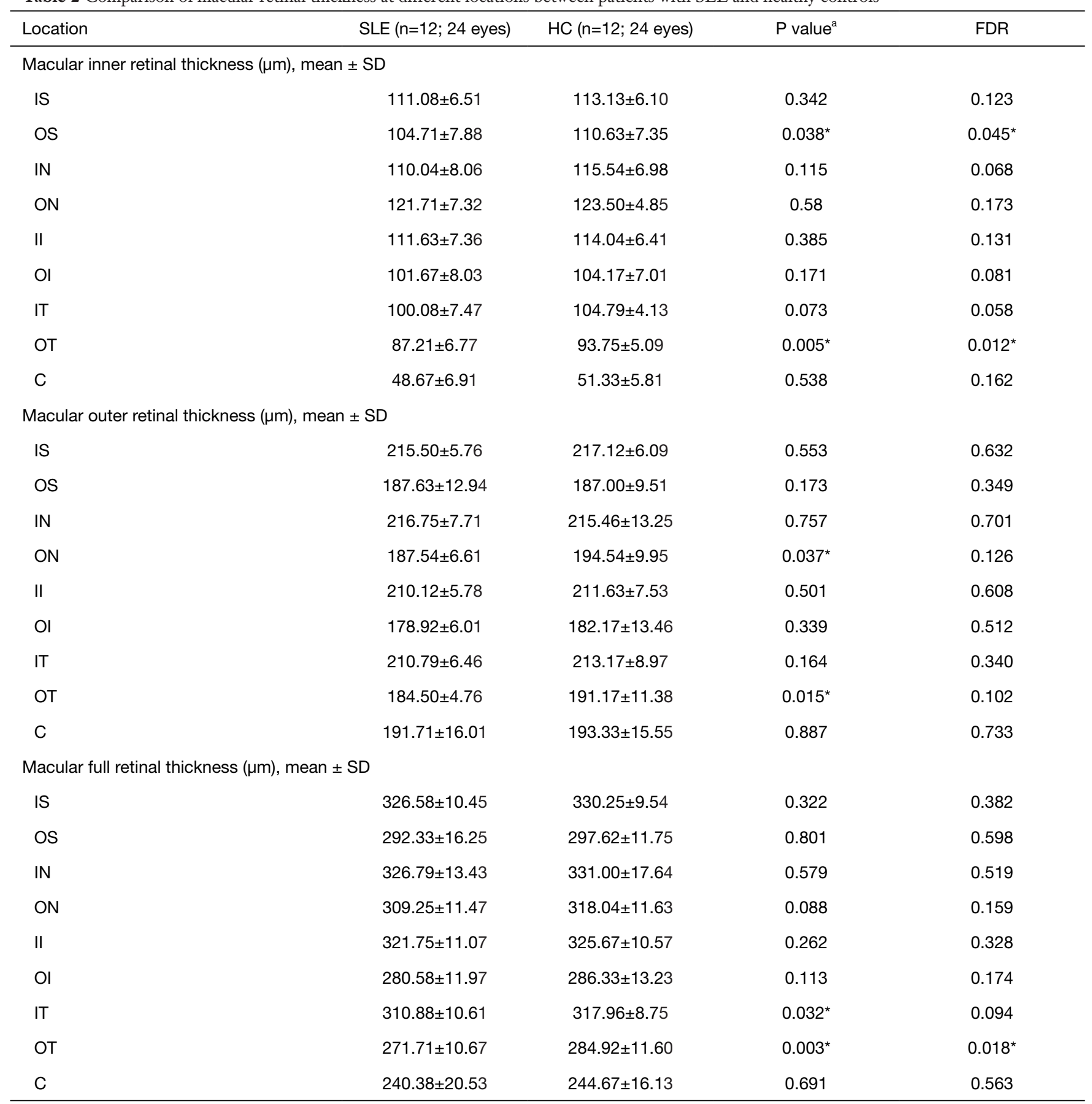

FDR was used to adjust $\mathrm{P}$ values. * $\mathrm{P}<0.05$; ${ }^{\text {, }}$, generalized estimating equation models were used to obtain $\mathrm{P}$ values comparing mean inner, outer, and full macular retinal thickness between SLE patients and healthy controls. Models were adjusted for age, gender, intraocular pressure, acuity, and blood pressure. SLE, systemic lupus erythematosus; HC, healthy control; SD, standard deviation; FDR, false discovery rate; IS, inner superior; OS, outer superior; IN, inner nasal; ON, outer nasal; II, inner inferior; OI, outer inferior; IT, inner temporal; OT, outer temporal; C center. 
Table 3 Univariate and multivariate regression analyses of association between macular retinal thickness with demographic and ocular parameters in patients with SLE

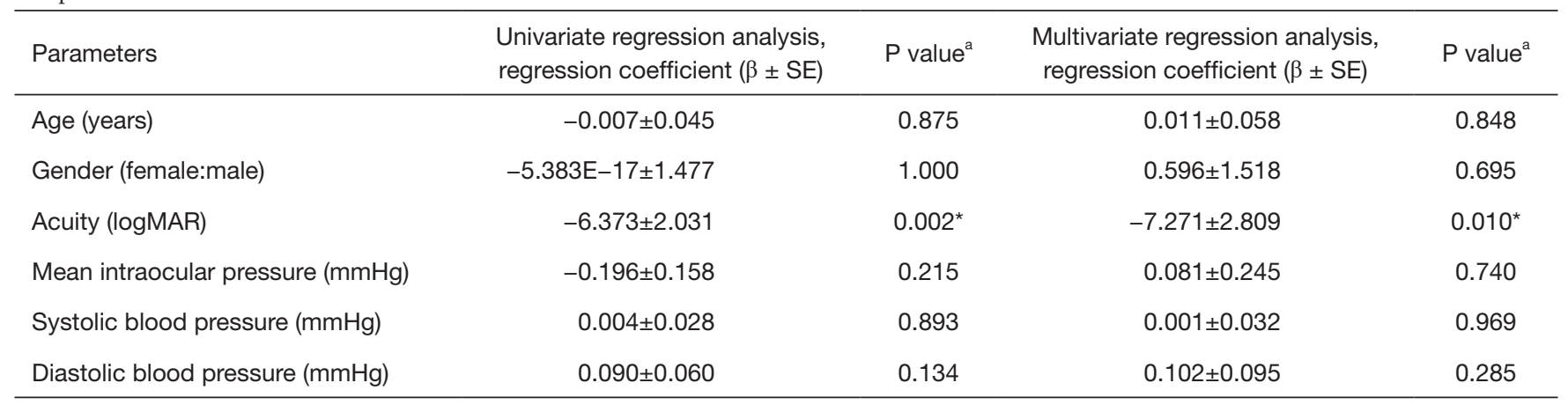

*, $\mathrm{P}<0.05 ;{ }^{a}$, $\mathrm{P}$ value was obtained with generalized estimating equation. SLE, systemic lupus erythematosus; SE, standard error; MAR, minimum angle of resolution.

Table 4 Comparison of superficial vessel density at different locations between patients with SLE and healthy participants

\begin{tabular}{|c|c|c|c|c|}
\hline Location $(\%$, mean \pm SD) & SLE $(n=12,24$ eyes $)$ & $\mathrm{HC}(\mathrm{n}=12,24$ eyes) & $P$ value $^{a}$ & FDR \\
\hline OS & $51.54 \pm 3.59$ & $52.21 \pm 3.77$ & 0.096 & $0.013^{*}$ \\
\hline IN & $50.63 \pm 4.84$ & $53.12 \pm 4.08$ & $0.023^{*}$ & $0.004^{*}$ \\
\hline ON & $54.83 \pm 2.79$ & $55.92 \pm 2.57$ & $0.018^{\star}$ & $0.003^{*}$ \\
\hline OI & $50.58 \pm 3.68$ & $52.63 \pm 3.89$ & $0.007^{\star}$ & $0.003^{*}$ \\
\hline IT & $52.21 \pm 3.95$ & $54.92 \pm 2.55$ & $0.010^{*}$ & $0.003^{*}$ \\
\hline OT & $47.29 \pm 4.03$ & $50.67 \pm 2.84$ & $0.003^{*}$ & $0.002^{*}$ \\
\hline C & $17.46 \pm 5.29$ & $22.00 \pm 3.24$ & $0.004^{*}$ & $0.002^{*}$ \\
\hline
\end{tabular}

FDR was used to adjust $\mathrm{P}$ values. *, $\mathrm{P}<0.05$; ${ }^{\text {, }}$, generalized estimating equation models were used to obtain $\mathrm{P}$ values to compare mean superficial vessel density between SLE patients and healthy controls. Models were adjusted for age, gender, intraocular pressure, acuity, and blood pressure. SD, standard deviation; SLE, systemic lupus erythematosus; HC, healthy control; FDR, false discovery rate; IS, inner superior; OS, outer superior; IN, inner nasal; ON, outer nasal; II, inner inferior; OI, outer inferior; IT, inner temporal; OT, outer temporal; C center.

diagnosis of SLE. However, ocular and visual factors in the assessment of this disease are often ignored. We found that in the absence of clear ocular symptoms, SLE participants had significantly poorer visual acuity than did the healthy controls, which has rarely been reported in previous studies.

Although ocular symptoms are not part of the diagnostic criteria for SLE, ocular manifestations remain important clues to disease activity since they provide direct visualization of vascular changes (26). The retina and the brain originate from the same embryological tissue and provide clues for the study of systemic diseases that may involve the CNS (34). Reduced RT may be the earliest sign of neurological disease and may help distinguish abnormality (17). In our study, according to the ETDRS zoning method, the RT was reduced in all regions, with significant thinning in OS and OT in the inner retina and OT in the full-thickness retina. A multivariate regression model showed that impaired vision was significantly associated with retinal thinning. This is consistent with previous studies showing retinal nerve fiber layer thinning in SLE $(23,24)$. OCTA measurements of the full RT in SLE have not been reported previously.

In SLE, subclinical infiltration caused by high permeability and increased choroidal thickness may induce choroidal lesions (35). Choroidal lesions can therefore indicate onset of disease months in advance of symptoms, are signals for subclinical reversible kidney disease and 

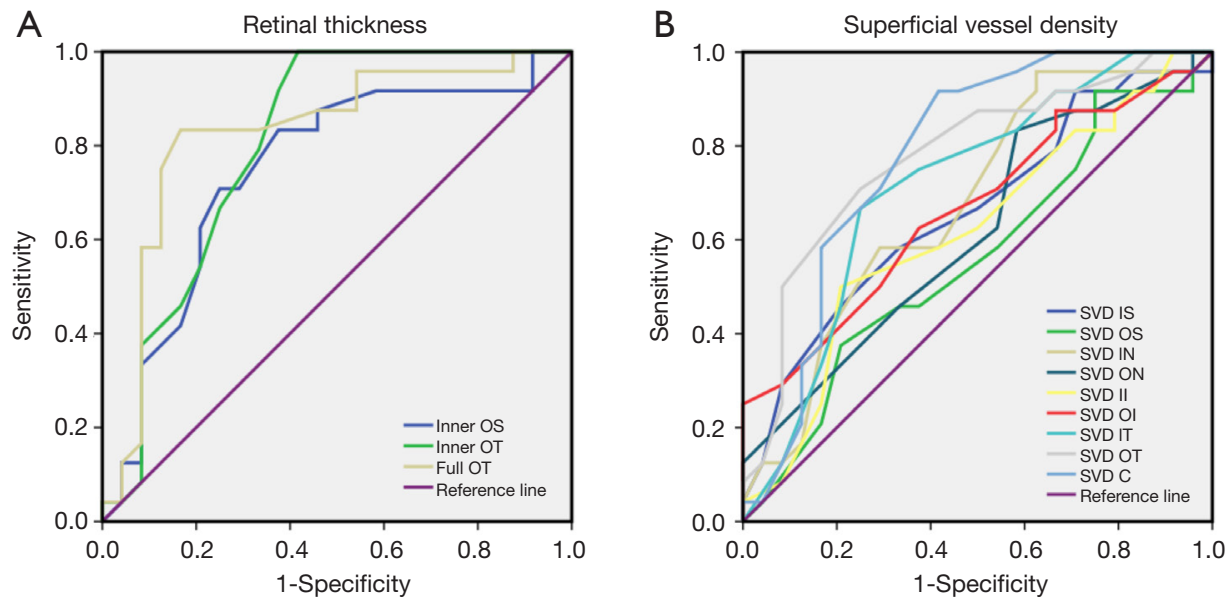

Figure 2 ROC curve analysis of RT and SVD. (A) The area under the ROC curve was 0.749 (95\% CI: 0.605 to 0.894 ) for inner OS, 0.805 for inner OT (95\% CI: 0.674 to 0.935 ), and 0.828 for full OT (95\% CI: 0.701 to 0.955 ). (B) The area under the ROC curve was 0.661 (95\% CI: 0.506 to 0.815 ) for SVD IS, 0.563 for SVD OS (95\% CI: 0.398 to 0.727 ), 0.677 for SVD IN (95\% CI: 0.524 to 0.831 ), 0.618 for SVD ON (95\% CI: 0.459 to 0.777$), 0.618$ for SVD II (95\% CI: 0.457 to 0.779$), 0.664$ for SVD OI (95\% CI: 0.510 to 0.818 ), 0.715 for SVD IT (95\% CI: 0.567 to 0.864 ), 0.779 for SVD OT (95\% CI: 0.644 to 0.913 ), and 0.780 for SVD C (95\% CI: 0.643 to 0.916 ). ROC, receiver operating characteristic; RT, retinal thickness; SVD, superficial vessel density; OS, outer superior; OT, outer temporal; ON, outer nasal; IT, inner temporal; IS, inner superior; IN, inner nasal; II, inner inferior; OI, outer inferior; C, center.
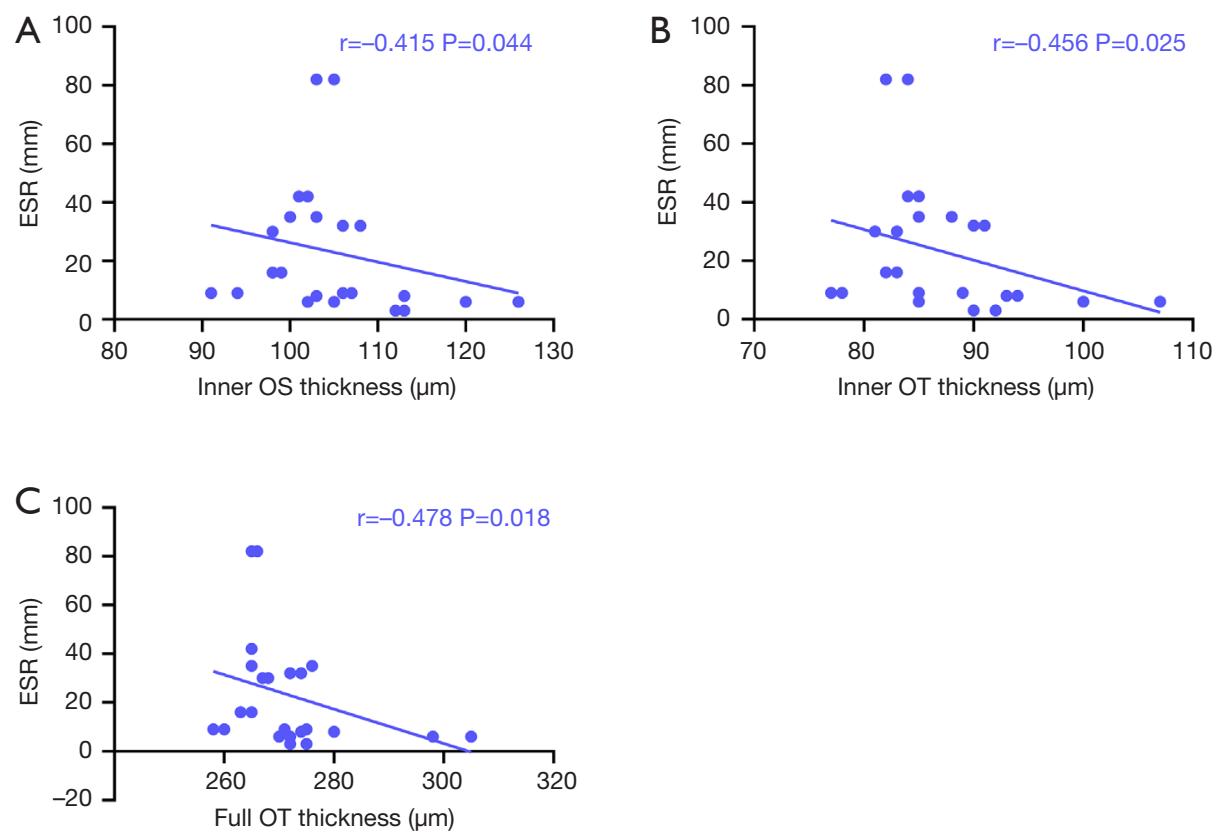

Figure 3 The correlation between RT and ESR in control and SLE patients. The vertical coordinate is the value of ESR, and the horizontal coordinate is the value of RT. (A) A negative correlation was found between RT and ESR in the inner retina in the OS region ( $\mathrm{r}=-0.415$; $\mathrm{P}=0.044)$. (B) A negative correlation was found between RT and ESR in the inner retina at OT region $(\mathrm{r}=-0.456$; $\mathrm{P}=0.025)$. $(\mathrm{C}) \mathrm{A}$ negative correlation was found between RT and ESR in the full retina in the OT region ( $\mathrm{r}=-0.478 ; \mathrm{P}=0.018)$. SLE, systemic lupus erythematosus; RT, retinal thickness; ESR, erythrocyte sedimentation rate; OS, outer superior; OT, outer temporal. 
A

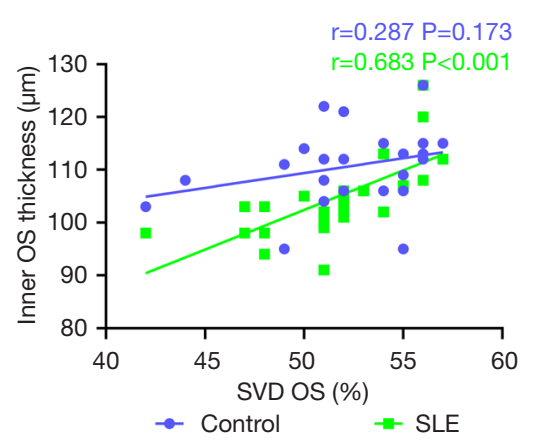

C

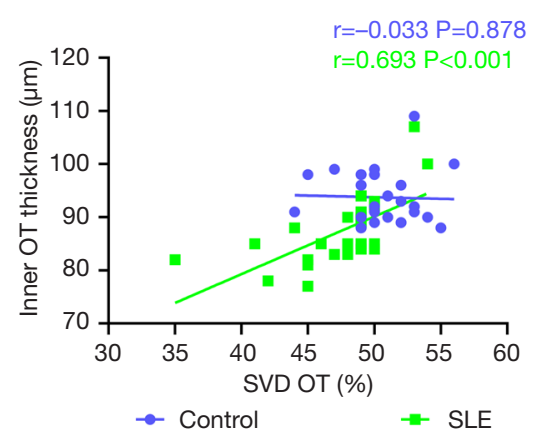

E

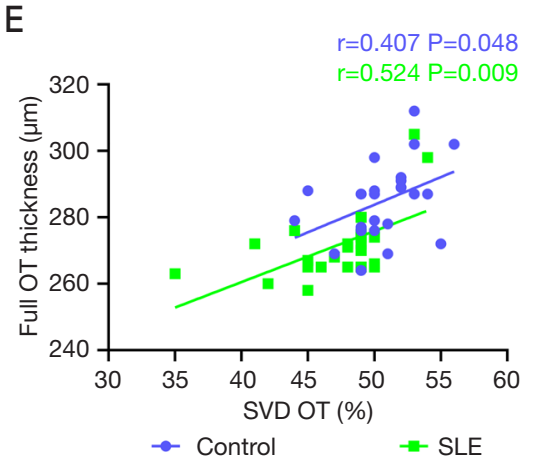

B

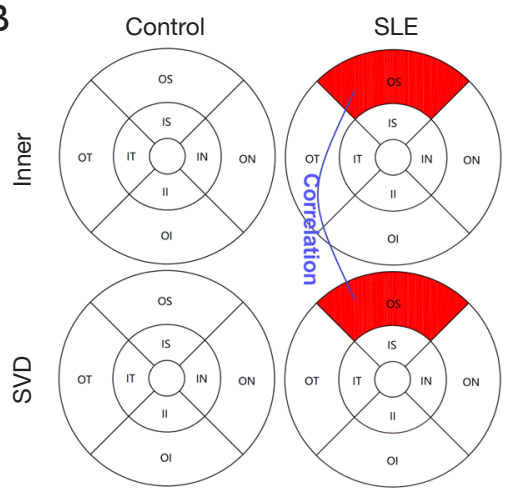

D

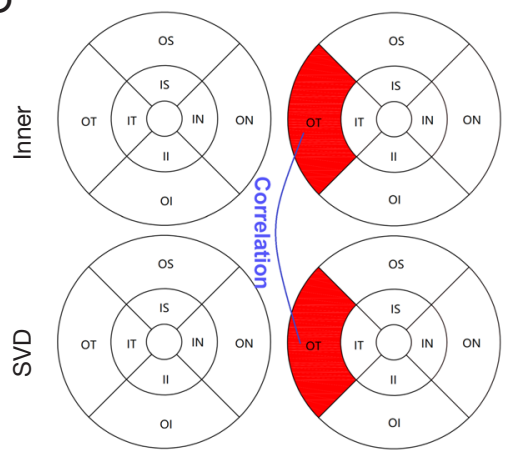

$\mathrm{F}$

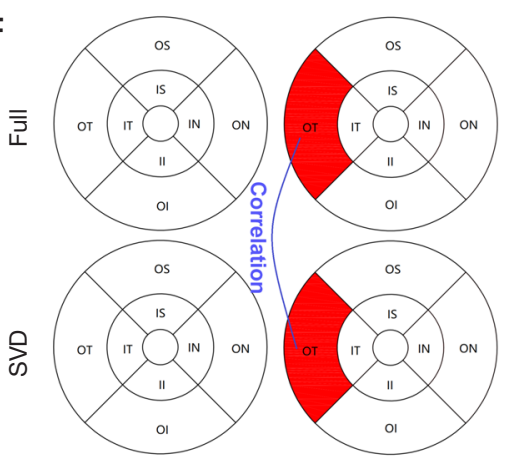

Figure 4 Correlation between RT and SVD in control and SLE patients. The vertical coordinate is the value of RT, and the horizontal coordinate is the value of SVD. (A,B) In the SLE group, SVD was positively correlated with inner RT in the OS region $(r=0.683 ; \mathrm{P}<0.001)$. (C,D) In the SLE group, SVD was positively correlated with inner RT in the OT region ( $r=0.693 ; \mathrm{P}<0.001)$. (E,F) In the SLE group, SVD was positively correlated with full RT in the OT region ( $r=0.524 ; \mathrm{P}=0.009)$. SLE, systemic lupus erythematosus; RT, retinal thickness; SVD, superficial vessel density; OS, outer superior; OT, outer temporal.

neuropathy (8), and are thus monitored by clinicians. If subclinical change of this kind also exists in the retina, it may prove similarly helpful.

The retina receives nutrition from three sources: central retinal blood vessels feed the inner layers, choroidal vessels (ciliary vascular system) feed the outer layers, while the macular region is served by choroidal capillaries. Patients with SLE have immune disorder, produce autoantibodies, and have abnormal activation of $\mathrm{T}$ and $\mathrm{B}$ lymphocytes (36). When the choroidal vascular system is involved, fibrin deposition in the vascular wall and lumen may occur (37), leading to vascular injury and increased vascular permeability, confirmed by histopathology. In this situation, inflammatory cell infiltration of the choroid, IgG (28) and 


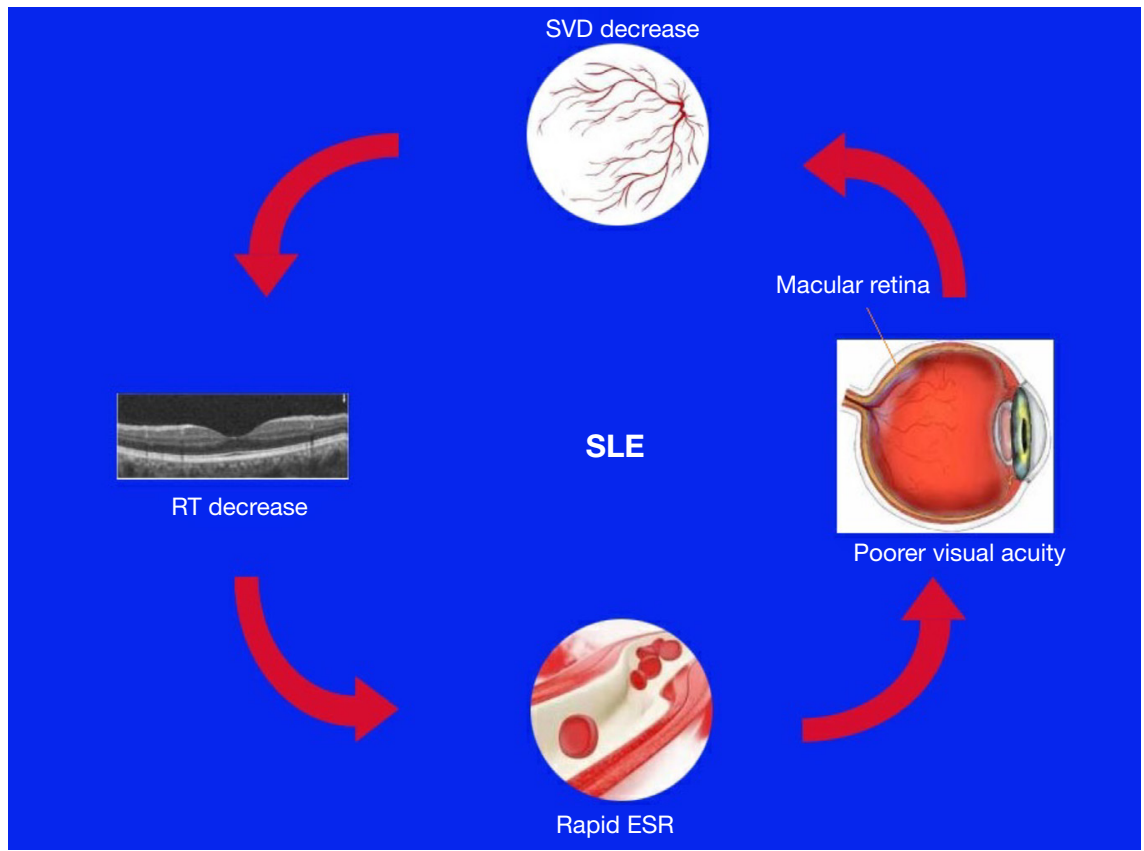

Figure 5 Relationship between retinal changes and microvessels in patients with SLE. The decrease of SVD led to the decrease of RT and visual acuity, which was related to a rapid progression of ESR. SLE, systemic lupus erythematosus; RT, retinal thickness; SVD, superficial vessel density; ESR, erythrocyte sedimentation rate.

complement deposition in the choroidal vascular system, and retinal pigment epithelial damage have been found (38). Antiphospholipid antibodies can also cause vascular lesions $(8,39)$, mainly due to the activation of platelet and coagulation pathways, leading to the formation of vascular microthrombosis (36), with the subsequent lumen stenosis resulting in tissue hypoxia and chronic ischemia $(40,41)$. When these changes occur in vessels supplying the retina, pathology of the retinal region follows, and these pathological changes may affect the whole chorioretinal vascular network. Our study found that in SLE patients, the superficial retinal vascular density decreased significantly in all regions. The inner RT and the full RT were positively associated with the SVD. These changes and correlations have also been found in diabetic patients (42). The microcirculation changes in diabetic patients may occur before clinically distinguishable retinopathy, and we speculate that this subclinical change also exists in SLE patients. Retinal thinning was most significant in the temporal regions, while the loss of capillaries occurred in all areas. Vascular lesions in the choroidal capillaries could also lead to long-term ischemia in the outer retina, especially in the photoreceptor layer, which would increase the production of reactive oxygen species. This detrimental environment reduces mitochondrial biosynthesis, resulting in inadequate energy supply and degeneration of photoreceptors in patients with SLE (43), thus affecting vision. Perhaps due to the limited sample size, we did not detect any significant difference in the thickness of outer retina between SLE patients and controls.

In SLE patients, many immune complexes are deposited in the capillaries to activate the complement system, and C5 is activated to produce C5a. The latter chemotactically stimulates neutrophils and other inflammatory cells to release more inflammatory factors, which clears the complex, aggravates the inflammatory response, and damages multiple organs and tissues (44). The immune regulation function of SLE patients is disordered, releasing numerous inflammatory factors $(45,46)$ to stimulate the aggregation of red blood cells, resulting in decreased stability and increased ESR. In the present study, thinning of the inner retina at the OS and OT regions and of the full retina at the OT were associated with elevated ESR. This reflects systemic inflammation and may serve as a marker of ineffective systemic disease control $(1,47)$ (Figure 5). CRP is also a nonspecific inflammation marker, which is often used to reflect the degree of inflammation in SLE patients, just like ESR; however, in our study, there was 
no correlation between RT and CRP. Variation in CRP values across different types of organ involvement in SLE has been proposed. The level of CRP in patients with lung involvement was significantly higher than that in patients with other types of organ involvement (48). Moreover, CRP level was positively correlated with the severity of pulmonary infection (49), especially bacterial infection (50). It has been reported that infected-inactive SLE has a significantly elevated CRP level in comparison with noninfected-inactive SLE (51).

Our ROC curve analysis of inner and full RT in the OT zone illustrates a possible approach for early detection of retinal changes in SLE. Early diagnosis and assessment are key to successful treatment and good prognosis. OCTA is a noninvasive, convenient measurement which provides information about intraocular vascular network perfusion. Changes in microcirculation in patients with SLE may precede clinically distinguishable retinopathy. OCTA is a useful method for differentiating healthy eyes from those with SLE. The RT measured by OCTA may be helpful for the assisted imaging diagnosis of SLE and an indicator of its inflammatory activity. However, more research is needed to provide a basis for future clinical application. Therefore, given the significant results of this study, it is necessary to include more participants in future studies.

A few other shortcomings in this research should be mentioned. First, patients with HCQ toxicity-related retinochoroidopathy were excluded, so the usage of HCQ in SLE patients was not comprehensively considered in this study. Previous research has shown that toxic effects of HCQ are not significant at a daily dose below $6.5 \mathrm{mg} / \mathrm{kg}$ over 5 years $(52,53)$. The doses and durations of HCQ used in the SLE participants included in this study were within the guideline-recommended range. Second, other drugs commonly used in SLE (including corticosteroids) may affect vascular integrity, yet these were not included in the scope of our research. In the context of SLE, we think that the influence of various autoantibodies and circulating immune complexes on blood vessels are the most important considerations. Glucocorticoids are one of the most important therapeutic drugs. They can inhibit the vasodilation of arterioles and capillary beds, thus preventing the increase of blood flow in the early stage of inflammation (54). They also prevent capillary and retrocapillary venules from increasing vascular permeability, thereby reducing the formation of exudates. At the same time, glucocorticoids can effectively inhibit the migration of leukocytes through the posterior venules of capillaries (55). In SLE, long-term administration of glucocorticoids almost inevitably results in a well-known series of adverse effects, including diabetes, osteoporosis, and fractures (56). Ocular adverse reactions mainly manifest as cataracts under the posterior capsule and as secondary open angle glaucoma (57). It has been reported that glucocorticoids are the main treatment methods, even in isolated retinal vasculitis (58). In our study, the decrease of RT and SVD in SLE patients was more likely to be caused by systemic diseases, and glucocorticoids should play a protective role. Adverse reactions of other drugs, including glucocorticoids, should be taken into account, but this needs further research, and may be the direction of our next study. Finally, the study included a small sample, and there were not enough participants to break down the comparison by disease stage. Further research with larger numbers of participants with SLE is needed in future. A larger participant cohort is required to confirm the present findings and provide a firmer basis for transition to clinical applications.

\section{Conclusions}

We used OCTA to enhance the understanding of RT and SVD in patients with SLE. The results included thinning of the inner and full thickness retina, SVD decrease in all areas, and a positive association between changes in RT with SVD. In addition, retinal thinning at the macular area was found to affect visual acuity, measurement of which by OCTA may be helpful for the assisted imaging diagnosis of SLE and an indicator of its inflammatory activity.

\section{Acknowledgments}

Funding: This research was supported by The Central Government Guides Local Science and Technology Development Foundation (No. 20211ZDG02003), the Key Research Foundation of Jiangxi Province (No. 20181BBG70004), the Excellent Talents Development Project of Jiangxi Province (No. 20192BCBL23020), the Natural Science Foundation of Jiangxi Province (No. 20181BAB205034), the Grassroots Health Appropriate Technology "Spark Promotion Plan" Project of Jiangxi Province (No. 20188003), the Health Development Planning Commission Science Foundation of Jiangxi Province (No. 20201032), and the Health Development Planning Commission Science TCM Foundation of Jiangxi Province (No. 2018A060). 


\section{Footnote}

Conflicts of Interest: All authors have completed the ICMJE uniform disclosure form (available at https://dx.doi. org/10.21037/qims-21-359). The authors have no conflicts of interest to declare.

Ethical Statement: The authors are accountable for all aspects of the work in ensuring that questions related to the accuracy or integrity of any part of the work are appropriately investigated and resolved. All research methods were approved by the Medical Ethics Committee of the First Affiliated Hospital of Nanchang University (cdyfy2016023) and were conducted in accordance with the Declaration of Helsinki (as revised in 2013). All participants were explained the purpose, method, and potential risks of the study, and provided written informed consent.

Open Access Statement: This is an Open Access article distributed in accordance with the Creative Commons Attribution-NonCommercial-NoDerivs 4.0 International License (CC BY-NC-ND 4.0), which permits the noncommercial replication and distribution of the article with the strict proviso that no changes or edits are made and the original work is properly cited (including links to both the formal publication through the relevant DOI and the license). See: https://creativecommons.org/licenses/by-nc-nd/4.0/.

\section{References}

1. Silpa-archa S, Lee JJ, Foster CS. Ocular manifestations in systemic lupus erythematosus. Br J Ophthalmol 2016;100:135-41.

2. Tsokos GC. Systemic lupus erythematosus. N Engl J Med 2011;365:2110-21.

3. Read RW. Clinical mini-review: systemic lupus erythematosus and the eye. Ocul Immunol Inflamm 2004;12:87-99.

4. Pons-Estel GJ, Alarcón GS, Scofield L, Reinlib L, Cooper GS. Understanding the epidemiology and progression of systemic lupus erythematosus. Semin Arthritis Rheum 2010;39:257-68.

5. Rees F, Doherty M, Grainge MJ, Lanyon P, Zhang W. The worldwide incidence and prevalence of systemic lupus erythematosus: a systematic review of epidemiological studies. Rheumatology (Oxford) 2017;56:1945-61.

6. Tedeschi SK, Johnson SR, Boumpas D, Daikh D, Dörner T, Jayne D, Kamen D, Lerstrøm K, Mosca M,
Ramsey-Goldman R, Sinnette C, Wofsy D, Smolen JS, Naden RP, Aringer M, Costenbader KH. Developing and Refining New Candidate Criteria for Systemic Lupus Erythematosus Classification: An International Collaboration. Arthritis Care Res (Hoboken) 2018;70:571-81.

7. Palejwala NV, Walia HS, Yeh S. Ocular manifestations of systemic lupus erythematosus: a review of the literature. Autoimmune Dis 2012;2012:290898.

8. Nguyen QD, Uy HS, Akpek EK, Harper SL, Zacks DN, Foster CS. Choroidopathy of systemic lupus erythematosus. Lupus 2000;9:288-98.

9. Mukherjee B, Nair AG. Principles and practice of external digital photography in ophthalmology. Indian J Ophthalmol 2012;60:119-25.

10. Do DV, Gower EW, Cassard SD, Boyer D, Bressler NM, Bressler SB, Heier JS, Jefferys JL, Singerman LJ, Solomon SD. Detection of new-onset choroidal neovascularization using optical coherence tomography: the AMD DOC Study. Ophthalmology 2012;119:771-8.

11. Do DV. Detection of new-onset choroidal neovascularization. Curr Opin Ophthalmol 2013;24:244-7.

12. Matsunaga D, Yi J, Puliafito CA, Kashani AH. OCT angiography in healthy human subjects. Ophthalmic Surg Lasers Imaging Retina 2014;45:510-5.

13. Ghasemi Falavarjani K, Tian JJ, Akil H, Garcia GA, Sadda SR, Sadun AA. Swept-source optical coherence tomography angiography of the optic disk in optic neuropathy. Retina 2016;36 Suppl 1:S168-77.

14. Spaide RF, Klancnik JM Jr, Cooney MJ. Retinal vascular layers imaged by fluorescein angiography and optical coherence tomography angiography. JAMA Ophthalmol 2015;133:45-50.

15. Spaide RF, Fujimoto JG, Waheed NK, Sadda SR, Staurenghi G. Optical coherence tomography angiography. Prog Retin Eye Res 2018;64:1-55.

16. Wu H, Sekiryu T, Sugano Y, Itagaki K, Kasai A, Shintake $\mathrm{H}$. A modified measuring method to investigate the choriocapillaris flow void of polypoidal choroidal vasculopathy with swept source optical coherence tomography angiography. Quant Imaging Med Surg 2021;11:3146-56.

17. Chhablani PP, Ambiya V, Nair AG, Bondalapati S, Chhablani J. Retinal Findings on OCT in Systemic Conditions. Semin Ophthalmol 2018;33:525-46.

18. Cunha JP, Proença R, Dias-Santos A, Almeida R, Águas H, Alves M, Papoila AL, Louro C, Castanheira-Dinis A. OCT in Alzheimer's disease: thinning of the RNFL and 
superior hemiretina. Graefes Arch Clin Exp Ophthalmol 2017;255:1827-35.

19. Durbin MK, An L, Shemonski ND, Soares M, Santos T, Lopes M, Neves C, Cunha-Vaz J. Quantification of Retinal Microvascular Density in Optical Coherence Tomographic Angiography Images in Diabetic Retinopathy. JAMA Ophthalmol 2017;135:370-6.

20. Lai FHP, Iao TWU, Ng DSC, Young AL, Leung J, Au A, Ko STC, Chong KKL. Choroidal thickness in thyroid-associated orbitopathy. Clin Exp Ophthalmol 2019;47:918-24.

21. Inzelberg R, Ramirez JA, Nisipeanu P, Ophir A. Retinal nerve fiber layer thinning in Parkinson disease. Vision Res 2004;44:2793-7.

22. Gordon-Lipkin E, Chodkowski B, Reich DS, Smith SA, Pulicken M, Balcer LJ, Frohman EM, Cutter G, Calabresi PA. Retinal nerve fiber layer is associated with brain atrophy in multiple sclerosis. Neurology 2007;69:1603-9.

23. Liu GY, Utset TO, Bernard JT. Retinal nerve fiber layer and macular thinning in systemic lupus erythematosus: an optical coherence tomography study comparing SLE and neuropsychiatric SLE. Lupus 2015;24:1169-76.

24. Dias-Santos A, Tavares Ferreira J, Pinheiro S, Cunha JP, Alves M, Papoila AL, Moraes-Fontes MF, Proença R. Neurodegeneration in systemic lupus erythematosus: layer by layer retinal study using optical coherence tomography. Int J Retina Vitreous 2020;6:15.

25. Ramage AE, Fox PT, Brey RL, Narayana S, Cykowski MD, Naqibuddin M, Sampedro M, Holliday SL, Franklin C, Wallace DJ, Weisman MH, Petri M. Neuroimaging evidence of white matter inflammation in newly diagnosed systemic lupus erythematosus. Arthritis Rheum 2011;63:3048-57.

26. Md Noh UK, Zahidin AZ, Yong TK. Retinal vasculitis in systemic lupus erythematosus: an indication of active disease. Clin Pract 2012;2:e54.

27. Shulman S, Shorer R, Wollman J, Dotan G, Paran D. Retinal nerve fiber layer thickness and neuropsychiatric manifestations in systemic lupus erythematosus. Lupus 2017;26:1420-5.

28. Karpik AG, Schwartz MM, Dickey LE, Streeten BW, Roberts JL. Ocular immune reactants in patients dying with systemic lupus erythematosus. Clin Immunol Immunopathol 1985;35:295-312.

29. Hochberg MC. Updating the American College of Rheumatology revised criteria for the classification of systemic lupus erythematosus. Arthritis Rheum 1997;40:1725.
30. Gladman DD, Ibañez D, Urowitz MB. Systemic lupus erythematosus disease activity index 2000. J Rheumatol 2002;29:288-91.

31. Gladman DD, Goldsmith CH, Urowitz MB, Bacon P, Fortin P, Ginzler E, Gordon C, Hanly JG, Isenberg DA, Petri M, Nived O, Snaith M, Sturfelt G. The Systemic Lupus International Collaborating Clinics/American College of Rheumatology (SLICC/ACR) Damage Index for Systemic Lupus Erythematosus International Comparison. J Rheumatol 2000;27:373-6.

32. Kashani AH, Chen CL, Gahm JK, Zheng F, Richter GM, Rosenfeld PJ, Shi Y, Wang RK. Optical coherence tomography angiography: A comprehensive review of current methods and clinical applications. Prog Retin Eye Res 2017;60:66-100.

33. Relhan N, Flynn HW Jr. The Early Treatment Diabetic Retinopathy Study historical review and relevance to today's management of diabetic macular edema. Curr Opin Ophthalmol 2017;28:205-12.

34. Karadag AS, Bilgin B, Soylu MB. Comparison of optical coherence tomographic findings between Behcet disease patients with and without ocular involvement and healthy subjects. Arq Bras Oftalmol 2017;80:69-73.

35. Maruko I, Iida T, Sugano Y, Ojima A, Sekiryu T. Subfoveal choroidal thickness in fellow eyes of patients with central serous chorioretinopathy. Retina 2011;31:1603-8.

36. Steiner M, Esteban-Ortega MDM, Muñoz-Fernández S. Choroidal and retinal thickness in systemic autoimmune and inflammatory diseases: A review. Surv Ophthalmol 2019;64:757-69.

37. Nag TC, Wadhwa S. Vascular changes of the retina and choroid in systemic lupus erythematosus: pathology and pathogenesis. Curr Neurovasc Res 2006;3:159-68.

38. Hannouche D, Korobelnik JF, Cochereau I, Hayem G, Beaudreuil J, Meyer O, Hoang-Xuan T. Systemic lupus erythematosus with choroidopathy and serous retinal detachment. Int Ophthalmol 1995;19:125-7.

39. Makino S, Tampo H. Rare and unusual choroidal abnormalities in a patient with systemic lupus erythematosus. Case Rep Ophthalmol 2013;4:81-6.

40. Skaggs BJ, Hahn BH, McMahon M. Accelerated atherosclerosis in patients with SLE--mechanisms and management. Nat Rev Rheumatol 2012;8:214-23.

41. Ferreira CS, Beato J, Falcão MS, Brandão E, Falcão-Reis F, Carneiro ÂM. Choroidal thickness in multisystemic autoimmune diseases without ophthalmologic manifestations. Retina 2017;37:529-35.

42. Kim K, Kim ES, Kim DG, Yu SY. Progressive retinal 
neurodegeneration and microvascular change in diabetic retinopathy: longitudinal study using OCT angiography. Acta Diabetol 2019;56:1275-82.

43. Sankowski R, Mader S, Valdés-Ferrer SI. Systemic inflammation and the brain: novel roles of genetic, molecular, and environmental cues as drivers of neurodegeneration. Front Cell Neurosci 2015;9:28.

44. Sadik CD, Miyabe Y, Sezin T, Luster AD. The critical role of $\mathrm{C} 5 \mathrm{a}$ as an initiator of neutrophil-mediated autoimmune inflammation of the joint and skin. Semin Immunol 2018;37:21-9.

45. Kamal A, Khamashta M. The efficacy of novel B cell biologics as the future of SLE treatment: a review. Autoimmun Rev 2014;13:1094-101.

46. Konya C, Paz Z, Tsokos GC. The role of T cells in systemic lupus erythematosus: an update. Curr Opin Rheumatol 2014;26:493-501.

47. Gallagher K, Viswanathan A, Okhravi N. Association of systemic lupus erythematosus with uveitis. JAMA Ophthalmol 2015;133:1190-3.

48. Firooz N, Albert DA, Wallace DJ, Ishimori M, Berel $\mathrm{D}$, Weisman $\mathrm{MH}$. High-sensitivity C-reactive protein and erythrocyte sedimentation rate in systemic lupus erythematosus. Lupus 2011;20:588-97.

49. Gao J, Zhang L, Zhang X, Zhao SL, Liu SY, Xing LH. Levels of serum procalcitonin and C-reactive protein for evaluating pulmonary bacterial infection in patients with lupus erythematosus. J Huazhong Univ Sci Technolog Med Sci 2016;36:653-8.

50. Tejani NR, Chonmaitree T, Rassin DK, Howie VM, Owen MJ, Goldman AS. Use of C-reactive protein in

Cite this article as: Liu R, Wang Y, Xia Q, Xu T, Han T, Cai S, Luo SL, Wu R, Shao Y. Retinal thickness and microvascular alterations in the diagnosis of systemic lupus erythematosus: a new approach. Quant Imaging Med Surg 2022;12(1):823-837. doi: 10.21037/qims-21-359 differentiation between acute bacterial and viral otitis media. Pediatrics 1995;95:664-9.

51. Zhang CF, Xu R, Li MT, Zeng XF. A clinical analysis on fever of unknown origin in in-patients with systemic lupus erythematosus. Zhonghua Nei Ke Za Zhi 2017;56:295-7.

52. Melles RB, Marmor MF. The risk of toxic retinopathy in patients on long-term hydroxychloroquine therapy. JAMA Ophthalmol 2014;132:1453-60.

53. Marmor MF, Kellner U, Lai TY, Melles RB, Mieler WF; American Academy of Ophthalmology. Recommendations on Screening for Chloroquine and Hydroxychloroquine Retinopathy (2016 Revision). Ophthalmology 2016;123:1386-94.

54. Perretti M, Ahluwalia A. The microcirculation and inflammation: site of action for glucocorticoids. Microcirculation 2000;7:147-61.

55. Tsurufuji S, Sugio K, Takemasa F. The role of glucocorticoid receptor and gene expression in the anti-inflammatory action of dexamethasone. Nature 1979;280:408-10.

56. Apostolopoulos D, Morand EF. It hasn't gone away: the problem of glucocorticoid use in lupus remains. Rheumatology (Oxford) 2017;56:1114-22.

57. Fel A, Aslangul E, Le Jeunne C. Eye and corticosteroid's use. Presse Med 2012;41:414-21.

58. Pelegrín L, Hernández-Rodríguez J, Espinosa G, Llorenç V, Sainz-de-la-Maza M, Fontenla JR, Martínez JA, Cid MC, Adán A. Characterization of isolated retinal vasculitis. Analysis of a cohort from a single center and literature review. Autoimmun Rev 2017;16:237-43. 